\title{
Preventive Maintenance Model Analysis Based on Condition
}

\author{
Hongsheng $\mathrm{Su}$ \\ School of Automation and Electrical Engineering, Lanzhou Jiaotong University, \\ Lanzhou 730070, China \\ shsen@163.com
}

\begin{abstract}
To improve operation maintenance management levels and increase economic profits for repairable devices, condition based preventive maintenance (CBM) has been widely applied in industrial enterprises in recent years. Compared with traditional time based maintenance (TBM), CBM not only can ensure the production process more safety but also dramatically reduce the examining-maintenance cost, which can be thought as a proactive maintenance scheme, practically. In this paper, we firstly construct the full life cycle model with four-state of the devices, and then simplified it into three-state model based on some practical considerations. On the basis of it, the paper analyzes its reliability operation characteristics under diverse maintenance models such as the proactive maintenance based on CBM, and the proactive maintenance based on TBM, and the passive maintenance, and the redundant maintenance scheme, and computes its desired maintenance cost and the investment payback period. The computing results show that the investments payback period is the shortest of the proactive maintenance rule based on CBM, and the one based on TBM comes second, and the one based on passive maintenance comes third, and the one of the redundant management model is the inferior. The investigations show that proactive maintenance based on CBM is a very effective operation maintenance management mode for repairable devices, and possesses very broad applications prosperity.
\end{abstract}

Keywords: Repairable devices, preventive maintenance, proactive maintenance, passive maintenance, investment payback period

\section{Introduction}

For those systems with high security and high reliability, preventive maintenance (PM) plays an important role and can provide them an ideal servicing management mode, such as aerospace systems, nuclear power plant, railway traffic systems and etc., Traditionally, PM adopts the time-based maintenance (TBM), a time interval fixed maintenance mode, the characteristics of which is that the optimal detection cycle or inspection interval can not be altered once confirmed in advance, and so it may possibly generates the deviation in practical applications due to the lack of dynamics and timeliness so as to incur a larger security risk and unnecessary economic loss[1, 2]. In addition, for a majority of production equipments, the confirmation of detection cycle or inspection interval is a complex and difficult task [3]. To resolve the issue, a sequential policy is proposed where the PM is performed in a time interval $\tau$, but the $\tau$ varies with the age of the components [4]. In [5] a so-called outstanding servicerepairing plan is proposed that declares to reconstruct the service-repairing strategies according to the related knowledge of service-repairing plan, equipment status, and spare cells stock, etc., But unfortunately, the strategy is too perfect so as that no model 
and theoretical support is achieved. Hence, a dynamic control strategy with CBM based is proposed for PM inspection intervals in [6], which can implement timely control on it according to the true state of the devices, correspondingly, the dynamics and timeliness to control the checking item is improved so as to reduce the maintenance and checking cost and improve the security. The essence of the control on check rate is to modify the expected mean time to failure (MTTF) so as to reduce the possibility of the corrective maintenance interventions, and consequently, the check and maintenance cost are reduced dramatically. Clearly, CBM not only improves the MTTF of the devices, but also reduces the PM cost, and is an ideal maintenance mode. Although the increment of the number of PM checks times in the unit of time leads to a rising of expense, but reduces the necessity of corrective maintenance $(\mathrm{CM})$, more expensive than $\mathrm{PM}$, thus from long run, an effective thrift can be wined [7]. In the paper, we first apply the theory knowledge of stochastic process to establish the full life cycle four-state model of the equipment, and then simplify it as three-state model from some practical considerations. Based on the model, the paper analyzes its reliability operation characteristics under diverse modes such as the proactive maintenance, the passive maintenance, and the redundant maintenance, and computes its desired maintenance cost and the payback period of investments. The outcomes show that proactive maintenance based on CBM is a very effective operation maintenance management mode, and possesses very broad applications prosperity.

\section{Model Description}

To construct the life cycle model of repairable devices, the following assumptions requires to be done below.

Assumption1. The preventive maintenance, or the corrective maintenance, is independently expressed only using a state.

Assumption2. Whether at the operating or storage state, there are no devices the failures of which are no detectable.

Assumption3. Both the preventive maintenance and the corrective maintenance, the check and maintenance time of which follow arbitrary distribution, in addition to it any other transfer rates are constant.

Assumption4. Preventive maintenance can not change the natural failure rate of the equipments, i.e., the instantaneous failure rate after the equipment is repaired is same with one before repaired, and only the residual life is owned when it returns to work.

Assumption5. Preventive maintenance is perfect, i.e., if there are faults detected out during preventive maintenance, and then it would be able to get a timely repair.

Assumption6. Corrective maintenance is perfect, i.e., the equipment can restore to the original state after corrective maintenance.

Based on the above assumptions, the life cycle model of the complicated devices can be presented using the state transition diagram in Figure 1.

In Figure 1, the symbol $S_{i}$ expresses the state of the equipment, where the subscript $i \in$ [1-4] is applied to respectively express the equipment storage, work, preventive maintenance, and corrective maintenance of four kinds of states; and $\lambda_{1}$ expresses the storage rate of a functioning equipment; and $\lambda_{2}$ expresses the using rate of a stored equipment; and $v_{1}$ presents the failure rate of a stored equipment; and $v_{2}$ presents the failure rate of a functioning equipment; and $u_{1}$ presents the check rate of a stored equipment; and $u_{2}$ presents the check rate of a functioning equipment; and $\rho_{1}$ expresses the stored probability of the device after a preventive maintenance intervention; and $1-\rho_{1}$ expresses the used probability of the device after a preventive maintenance intervention; and $\rho_{2}$ expresses the stored probability of the 
device after a corrective maintenance intervention; and 1- $\rho_{2}$ expresses the used probability of the device after a corrective maintenance intervention; and $\mu_{1}$ expresses the repair risk rate function of the preventive maintenance; $\mu_{2}$ expresses the repair risk rate function of the corrective maintenance. The physical meaning of the transfer rate is to ensure that it is the bounded and the nonnegative. Let $S(t)$ be the situated state of the device at time instant $t$, and then the $S(t)$ is a continuous time Markov process, namely, at arbitrary time instant $t$, as the specific numerical values of $S(t)$ are given, and the operation law after the time instant $t$ of the process $S(t)$ has nothing to do with the history before $t$.

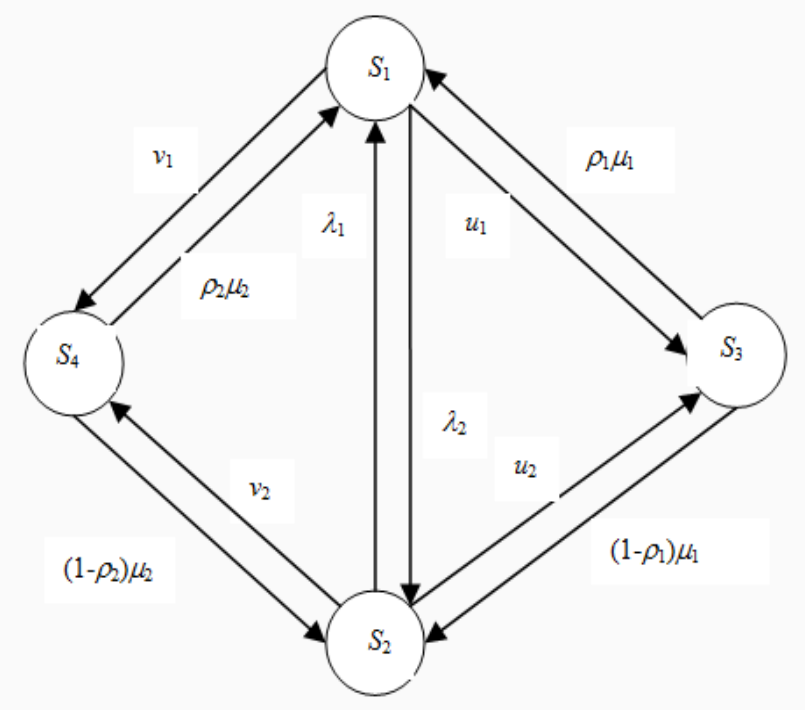

Figure 1. State Transition Diagram with Four-State

Based on model in Figure 1 and assumption 5 and 6, we further assume that PM can accomplish an eventual substitution before there is an upcoming failure or after a random damage happens, and thus the state $S_{4}$ can be eliminated from the life cycle model in Figure 1. And then, we will obtain the following model as shown in Figure 2.

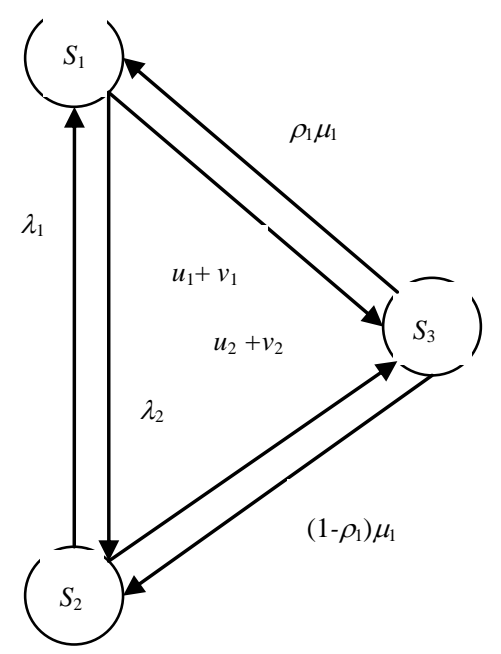

Figure 2. State Transition Diagram with Three-State 
The model in Figure 2 only represents the active life of the devices, the infant mortality is ignored, and obsolescence ageing is not included due to an eventual substitution under PM.

It is obvious that the process in Figure 2 possesses Markov.

For $\forall t \geq 0$, we use $x_{i}(t)$ to express the situated state of system $S(t)$ at time instant $t$, and then

$$
x_{i}(t)=p\left\{S(t)=S_{i}\right\}, \quad i=1,2,3 .
$$

Deinition1. The reliability degree of the equipment is defined as the probability that it can work normally within the scope of the time from zero to $t$. It does not allow failure, and so does not maintenance. According to the definition, the reliability degree $R(t)$ should have nothing to do with traversing what states before the device becomes the failure for the first time. In this system, since the natural failure or obsolescence due to ageing is eliminated, and moreover, based on consideration on assumption 4, we think although the preventive state $S_{3}$ could arouse machines halt, but not influence the equipments reliability. But when computing MTTF, the time that generator stays at the state $S_{3}$ should be deducted before it is possibly given an eventual substitution. Hence, according to [8,9], we have

$$
\begin{aligned}
R(t) & =P_{\mathrm{r}}(T>t)=1-\mathrm{P}_{\mathrm{r}}(T \leq t)=1-F(t)=1-\int_{0}^{t} f(t) \mathrm{d} t \\
& =\int_{t}^{\infty} f(t) \mathrm{d} t=\mathrm{F}(\infty)-\mathrm{F}(t)=R(t)-R(\infty) \\
& =x_{1}(t)+x_{2}(t)-x_{1}(\infty)-x_{2}(\infty)
\end{aligned}
$$

where $F(t)$ is the failure time distribution function, and whose probability density function $(p d f)$ is $f(t) t \geq 0$.

And then based on (2), MTTF can be defined by

$$
\mathrm{MTTF}=\int_{0}^{\infty} R(t) \mathrm{d} t=1^{\mathrm{T}} \int_{0}^{\infty}[\boldsymbol{x}(t)-\boldsymbol{x}(\infty)] \mathrm{d} t
$$

where $\mathbf{1}^{\mathrm{T}}=\left[\begin{array}{ll}1 & 1\end{array}\right]$, and $\boldsymbol{x}(t)=\left[\begin{array}{ll}x_{1}(t) & x_{2}(t)\end{array}\right]^{\mathrm{T}}$, and $\boldsymbol{x}(t) \boldsymbol{x}(\infty)=\left[\begin{array}{ll}x_{1}(\infty) & x_{2}(\infty)\end{array}\right]^{\mathrm{T}}$. Clearly, if $\boldsymbol{x}(\infty)=\mathbf{0}$, then the MTTF in traditional meaning is acquired.

\section{TBM Model Analysis}

TBM is a widely applied maintenance model in industrial enterprises, the characteristic is that the checking-repairing arrangement of the devices is performed based equivalent time interval, and also called as a kind of PM with the fixed time interval, where the fixed time interval is constant, i.e., the checking rates $u_{1}$ and $u_{2}$ are constants. In general, this maintenance means can find and resolve the majority of problems during devices operating, but the main problem of which is maintenance surplus and deficiency, and also is difficult task to determine the time interval [10]. Even so, TBM is still a broadly applied PM method, so far today it continues to be broadly used.

According to Figure 2 and reliability mathematics theory [11, 12] and we then have the following Kolmogorov equation group.

$$
\left\{\begin{array}{l}
\mathrm{d} x_{1}(t) / \mathrm{d} t=-\left(\lambda_{2}+u_{1}+v_{1}\right) x_{1}(t)+\lambda_{1} x_{2}(t)+\rho_{1} \mu_{1} x_{3}(t) \\
\mathrm{d} x_{2}(t) / \mathrm{d} t=\lambda_{2} x_{1}(t)-\left(\lambda_{1}+u_{2}+v_{2}\right) x_{2}(t)+\left(1-\rho_{1}\right) \mu_{1} x_{3}(t) \\
\mathrm{d} x_{3}(t) / \mathrm{d} t=\left(u_{1}+v_{1}\right) x_{1}(t)+\left(u_{2}+v_{2}\right) x_{2}(t)-\mu_{1} x_{3}(t)
\end{array}\right.
$$

The physical bounds of the transition rates satisfy

$$
\begin{aligned}
& 0 \leq \lambda_{1} \leq \lambda_{1 \max }, 0 \leq \lambda_{2} \leq \lambda_{2 \max }, 0 \leq v_{1} \leq v_{1 \max }, 0 \leq v_{2} \leq v_{2 \max }, 0 \leq u_{1} \leq u_{1 \max }, 0 \leq u_{2} \leq u_{2 \max }, \\
& 0 \leq \mu_{1} \leq \mu_{1 \max }, 0 \leq \rho_{1} \leq 1, \forall t \geq 0 .
\end{aligned}
$$

The system states are mutually exclusive, and therefore the following equation is bound to hold. 
where $0 \leq x_{1}(t) \leq 1,0 \leq x_{2}(t) \leq 1,0 \leq x_{3}(t) \leq 1, \forall t \geq 0$.

$$
x_{1}(t)+x_{2}(t)+x_{3}(t)=1, \quad \forall t \geq 0
$$

From (8), we can obtain

Substituting (9) into (4), and (5), we obtain

$$
x_{3}(t)=1-\left[x_{1}(t)+x_{2}(t)\right]
$$

$$
\left\{\begin{array}{l}
\mathrm{d} x_{1}(t) / \mathrm{d} t=-\left(\lambda_{2}+\lambda_{3}+\rho_{1} \mu_{1}\right) x_{1}(t)+\left(\lambda_{1}-\rho_{1} \mu_{1}\right) x_{2}(t)+\rho_{1} \mu_{1} \\
\mathrm{~d} x_{2}(t) / \mathrm{d} t=\left[\lambda_{2}-\left(1-\rho_{1}\right) \mu_{1}\right] x_{1}(t)-\left(\lambda_{1}+\lambda_{4}+\left(1-\rho_{1}\right) \mu_{1}\right) x_{2}(t)+\left(1-\rho_{1}\right) \mu_{1} \\
x_{1}(t)+x_{2}(t) \leq 1, \quad \forall t \geq 0
\end{array}\right.
$$

where $\lambda_{3}=u_{1}+v_{1}$ and $\lambda_{4}=u_{2}+v_{2}$.

Writting (10) and (11) into matrix form, and then

$$
\frac{\mathrm{d} \boldsymbol{x}(t)}{\mathrm{d} t}=\boldsymbol{A} \boldsymbol{x}(t)+\boldsymbol{\mu}
$$

where

$$
\begin{aligned}
& \boldsymbol{A}=\left[\begin{array}{cc}
-d_{1} & a \\
b & -d_{2}
\end{array}\right] \quad \boldsymbol{x}(t)=\left[\begin{array}{l}
x_{1}(t) \\
x_{2}(t)
\end{array}\right] \quad \boldsymbol{\mu}=\left[\begin{array}{l}
\rho_{1} \mu_{1} \\
\left(1-\rho_{1}\right) \mu_{1}
\end{array}\right] \\
& d_{1}=\lambda_{2}+\lambda_{3}+\rho_{1} \mu_{1}, a=\lambda_{1}-\rho_{1} \mu_{1}, d_{2}=\lambda_{1}+\lambda_{4}+\left(1-\rho_{1}\right) \mu_{1}, b=\lambda_{2}-\left(1-\rho_{1}\right) \mu_{1} .
\end{aligned}
$$

From (7), we can known that $\boldsymbol{A}$ is a stale matrix since the parameters of which satisfies $d_{1}>0$, $d_{2}>0, d_{1} d_{2}-a b>0$. In addition, it is natural that (7) also shows that $d_{1}-b>0, d_{2}-a>0, d_{1}+a>0$ and $d_{2}+b>0$. Hence, $\boldsymbol{A}$ is also a strong diagonally dominant matrix. Thus, according to the ergodicity characteristics of homogeneous Markov process, the system (13) is bound to possess solely invariable steady-state solution [13,14].

Theorem1. As the system described by (13) operating at steady-state, any initial states deviating from the steady states will return to the steady states, the required time of the return process respectively are, for $x_{1}(t), x_{2}(t)$

$$
\begin{aligned}
& \operatorname{MTTF}_{11}=\frac{d_{2}\left(x_{1}(0)-x_{1}(\infty)\right)+a\left(x_{2}(0)-x_{2}(\infty)\right)}{d_{1} d_{2}-a b} \\
& \operatorname{MTTF}_{21}=\frac{b\left(x_{1}(0)-x_{1}(\infty)\right)+d_{1}\left(x_{2}(0)-x_{2}(\infty)\right)}{d_{1} d_{2}-a b}
\end{aligned}
$$

Further, the total return time is

$$
\begin{aligned}
& \mathrm{MTTF}_{1}=\mathrm{MTTF}_{11}+\mathrm{MTTF}_{21} \\
& =\frac{\left(d_{2}+b\right)\left(x_{1}(0)-x_{1}(\infty)\right)+\left(a+d_{1}\right)\left(x_{2}(0)-x_{2}(\infty)\right)}{d_{1} d_{2}-a b}
\end{aligned}
$$

Proof. According to (13), we have

$$
\boldsymbol{x}(t)=e^{\boldsymbol{A} t}\left[\boldsymbol{x}(0)+\boldsymbol{A}^{-1} \boldsymbol{\mu}\right]-\boldsymbol{A}^{-1} \boldsymbol{\mu}
$$

Since $\boldsymbol{A}$ is a stable matrix, as $t \rightarrow \infty$ we have

$$
\boldsymbol{x}(\infty)=\left(\begin{array}{l}
x_{1}(\infty) \\
x_{2}(\infty)
\end{array}\right)=-\boldsymbol{A}^{-1} \boldsymbol{\mu}=\left(\begin{array}{l}
\frac{d_{2} \rho_{1} \mu_{1}+a\left(1-\rho_{1}\right) \mu_{1}}{d_{1} d_{2}-a b} \\
\frac{b \rho_{1} \mu_{1}+d_{1}\left(1-\rho_{1}\right) \mu_{1}}{d_{1} d_{2}-a b}
\end{array}\right)
$$

Hence, according to (9), we have 


$$
x_{3}(\infty)=1-\left[x_{1}(\infty)+x_{2}(\infty)\right]=\frac{\left(d_{1} d_{2}-a b\right)-\left[\left(d_{2}+b\right) \rho_{1} \mu_{1}+\left(a+d_{1}\right)\left(1-\rho_{1}\right) \mu_{1}\right]}{d_{1} d_{2}-a b}
$$

And then

$$
\boldsymbol{x}(t)-\boldsymbol{x}(\infty)=e^{A t}[\boldsymbol{x}(0)-\boldsymbol{x}(\infty)]
$$

Let $p_{1}$ and $p_{2}$ are the two by the eigenvalues of $\boldsymbol{A}$, the eigenvalues of the matrix $\boldsymbol{A}$ can be resolved by

$$
\begin{aligned}
& p_{1}=-\frac{d_{1}+d_{2}}{2}-\frac{\sqrt{\Delta}}{2} \\
& p_{2}=-\frac{d_{1}+d_{2}}{2}+\frac{\sqrt{\Delta}}{2}
\end{aligned}
$$

where

$$
\sqrt{\Delta}=\sqrt{\left(d_{1}-d_{2}\right)^{2}+4 a b}=p_{2}-p_{1}
$$

According to linear control theory [15], exp. $(\boldsymbol{A} t)$ can be solved by

$$
\exp (\boldsymbol{A} \boldsymbol{t})=\frac{1}{p_{2}-p_{1}}\left[\begin{array}{cc}
\left(p_{2}-d_{1}\right) e^{-p_{1} t}+\left(d_{1}-p_{1}\right) e^{-p_{2} t} & a\left(e^{-p_{1} t}-e^{-p_{2} t}\right) \\
b\left(e^{-p_{1} t}-e^{-p_{2} t}\right) & \left(p_{2}-d_{2}\right) e^{-p_{1} t}+\left(d_{2}-p_{1}\right) e^{-p_{2} t}
\end{array}\right]
$$

And so, we obtain

$$
\left[\begin{array}{l}
x_{1}(t)-x_{1}(\infty) \\
x_{2}(t)-x_{2}(\infty)
\end{array}\right]=\frac{1}{p_{2}-p_{1}}\left[\begin{array}{cc}
\left(p_{2}-d_{1}\right) e^{-p_{1} t}+\left(d_{1}-p_{1}\right) e^{-p_{2} t} & a\left(e^{-p_{1} t}-e^{-p_{2} t}\right) \\
b\left(e^{-p_{1} t}-e^{-p_{2} t}\right) & \left(p_{2}-d_{2}\right) e^{-p_{1} t}+\left(d_{2}-p_{1}\right) e^{-p_{2} t}
\end{array}\right]\left[\begin{array}{l}
x_{1}(0)-x_{1}(\infty) \\
x_{2}(0)-x_{2}(\infty)
\end{array}\right]
$$

According to (3), we have

$$
\begin{aligned}
& \operatorname{MTTF}_{11}=\int_{0}^{\infty}\left[x_{1}(t)-x_{1}(\infty)\right] \mathrm{d} t \\
& \operatorname{MTTF}_{21}=\int_{0}^{\infty}\left[x_{2}(t)-x_{2}(\infty)\right] \mathrm{d} t
\end{aligned}
$$

Substituting the first line and the second line of (21) into the above two formula, respectively, and (14) and (15) can be obtained, immediately.

In addition, according to (19) and (3), we have

$$
\begin{aligned}
\mathrm{MTTF}_{1} & =1^{\mathrm{T}} \int_{0}^{\infty}[\boldsymbol{x}(t)-\boldsymbol{x}(\infty)] \mathrm{d} t=1^{\mathrm{T}} \int_{0}^{\infty} e^{\boldsymbol{A} t}[\boldsymbol{x}(0)-\boldsymbol{x}(\infty)] \mathrm{d} t=-1^{\mathrm{T}} \boldsymbol{A}^{-1}[\boldsymbol{x}(0)-\boldsymbol{x}(\infty)] \\
& =\frac{\left(d_{2}+b\right)\left(x_{1}(0)-x_{1}(\infty)\right)+\left(a+d_{1}\right)\left(x_{2}(0)-x_{2}(\infty)\right)}{d_{1} d_{2}-a b}
\end{aligned}
$$

Clearly, it is easy to be validated that the formula (16) holds.

Theorem2 As the system described by (4) to (8) starting from steady-state, whose transient states deviating from the steady states under maintenance disturbance satisfy

$$
\Delta x_{1}(t)+\Delta x_{2}(t)+\Delta x_{3}(t)=0, \quad \forall t \geq 0
$$

where $\Delta x_{1}(t)=x_{1}(t)-x_{1}(\infty)$, and $\Delta x_{2}(t)=x_{2}(t)-x_{2}(\infty)$, and $\Delta x_{3}(t)=x_{3}(t)-x_{3}(\infty)$.

Under the condition of (22), any one of all the three states of $\Delta x_{1}(t), \Delta x_{2}(t)$, and $\Delta x_{3}(t)$ may act as the adjoint state, and the consumed time of which to reach the steady state equals the used time sum of another two states to arrive at their steady states.

Proof. In terms of the system described by (4) to (8), the steady probability of each state naturally meets 


$$
x_{1}(\infty)+x_{2}(\infty)+x_{3}(\infty)=1
$$

Under the condition of quite conservation, the probability of each state in transient process is also meets (8), and then

That is

$$
x_{1}(t)-x_{1}(\infty)+x_{2}(t)-x_{2}(\infty)+x_{3}(t)-x_{3}(\infty)=0
$$

$$
\Delta x_{1}(t)+\Delta x_{2}(t)+\Delta x_{3}(t)=0
$$

In (4), (5) and (6), we use $\Delta x_{1}(t), \Delta x_{2}(t)$, and $\Delta x_{3}(t)$ to replace the $x_{1}(t), x_{2}(t)$, and $x_{3}(t)$, respectively, and consider $\lambda_{3}=u_{1}+v_{1}$ and $\lambda_{4}=u_{2}+v_{2}$, and then

$$
\left[\begin{array}{l}
\frac{\mathrm{d} \Delta x_{1}(t)}{\mathrm{d} t} \\
\frac{\mathrm{d} \Delta x_{2}(t)}{\mathrm{d} t} \\
\frac{\mathrm{d} \Delta x_{3}(t)}{\mathrm{d} t}
\end{array}\right]=\left[\begin{array}{ccc}
-\left(\lambda_{2}+\lambda_{3}\right) & \lambda_{1} & \rho_{1} \mu_{1} \\
\lambda_{2} & -\left(\lambda_{1}+\lambda_{4}\right) & \left(1-\rho_{1}\right) \mu_{1} \\
\lambda_{3} & \lambda_{4} & -\mu_{1}
\end{array}\right]\left[\begin{array}{c}
\Delta x_{1}(t) \\
\Delta x_{2}(t) \\
\Delta x_{3}(t)
\end{array}\right]
$$

Writing the above equation as the matrix format, then

$$
\frac{\mathrm{d} \Delta \boldsymbol{x}(t)}{\mathrm{d} t}=\boldsymbol{B} \Delta \boldsymbol{x}(t)
$$

Clearly, $\operatorname{rank}(\boldsymbol{B})=2$, this indicates that the equations in (24) are of linear correlation, that is the anyone equation can be expressed by another two. We might as well define $\Delta x_{3}(t)$ as the adjoint state, that is the probability equals zero, or $x_{3}(t)=x_{3}(\infty)$ when it being in steady-steat. Substituting (22) into (24), we have

$$
\left[\begin{array}{l}
\frac{\mathrm{d} \Delta x_{1}(t)}{\mathrm{d} t} \\
\frac{\mathrm{d} \Delta x_{2}(t)}{\mathrm{d} t} \\
\frac{\mathrm{d} \Delta x_{3}(t)}{\mathrm{d} t}
\end{array}\right]=\left[\begin{array}{ccc}
-\left(\lambda_{2}+\lambda_{3}+\rho_{1} \mu_{1}\right) & \lambda_{1}-\rho_{1} \mu_{1} & 0 \\
\lambda_{2}-\left(1-\rho_{1}\right) \mu_{1} & -\left(\lambda_{1}+\lambda_{4}+\left(1-\rho_{1}\right) \mu_{1}\right) & 0 \\
\lambda_{3}+\mu_{1} & \lambda_{4}+\mu_{1} & 0
\end{array}\right]\left[\begin{array}{l}
\Delta x_{1}(t) \\
\Delta x_{2}(t) \\
\Delta x_{3}(t)
\end{array}\right]
$$

The equations show that the evolution process of $\Delta x_{1}(t), \Delta x_{2}(t)$, and $\Delta x_{3}(t)$ is determined by the changing process of the $\Delta x_{1}(t)$ and $\Delta x_{2}(t)$, that is, as the $\Delta x_{1}(t)$ and $\Delta x_{2}(t)$ arrive at steady states and end changes, and $\Delta x_{3}(t)$ also terminates its change. This means that $\Delta x_{3}(t)$ is an adjoint state. According to (26), we may plot the state transition diagram with the absorbing state as follows.

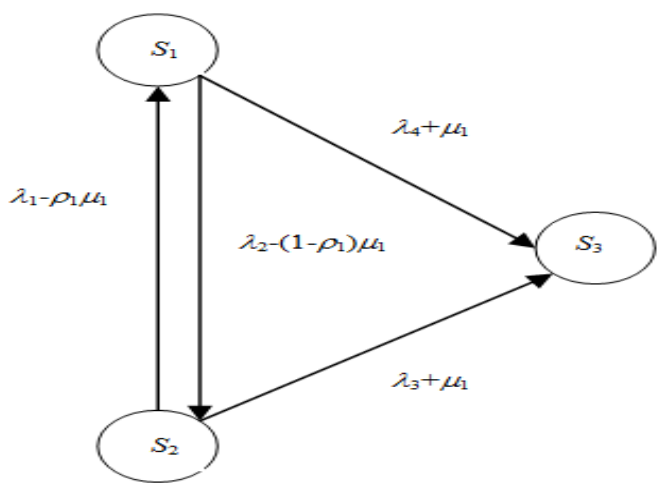

Figure 3. State Transition Diagram with Adjoint State 
As shown in Figure 3, $S_{3}$ is called as adjoint state, that is, as $S_{1}$ and $S_{2}$ stop changes, and $S_{3}$ also ends. That is to say, and the consumed time to reach the absorbing state equals the used time sum of another two states to arrive at their steady states. In fact, Theorem 1 also can explain the issue. (End)

Theorem3 As the system described by (4) to (8), and the expected steady-state time of each state can be estimated by

$$
\begin{aligned}
& \tau_{x 1(\infty)}=x_{2}(\infty) \mathrm{MTTF}_{21}+x_{3}(\infty) \mathrm{MTTF}_{1} \\
& \tau_{x 2(\infty)}=x_{1}(\infty) \mathrm{MTTF}_{11}+x_{3}(\infty) \mathrm{MTTF}_{1} \\
& \tau_{x 3(\infty)}=x_{1}(\infty) \mathrm{MTTF}_{11}+x_{2}(\infty) \mathrm{MTTF}_{21}
\end{aligned}
$$

In addition, the expected steady-state survival time is

$$
\tau_{1}=\tau_{x 1}(\infty)+\tau_{x 2}(\infty)+\tau_{x 3}(\infty)
$$

where $\tau_{x 1(\infty)}$ is the desired steady-state operating time of the state $x_{1}$, and $\tau_{x 2(\infty)}$ is the desired steady-state time of the state $x_{2}$, and $\tau_{x 3(\infty)}$ is the desired steady-state time of the state $x_{3}$.

Proof. According to Theorem2, for each device at steady-state, $\mathrm{MTTF}_{11}$ means the desired time spent waiting to enter into the warehouse, and $\mathrm{MTTF}_{21}$ means the desired time spent waiting to enter into functioning, and $\mathrm{MTTF}_{1}$ is the expected time spent waiting to enter into repairing. Let $\alpha$, and $\beta$, and $\gamma$ respectively be the real number with zero to one, and meet $\alpha+\beta+\gamma=1$.

And then

$$
\tau_{x 1(\infty)}=\alpha \mathrm{MTTF}_{21}+\beta \mathrm{MTTF}_{1}
$$

The two items at right side of the above formula represents the total expected time being ready to enter into the state two and state three for the device being in state one. In range of the time, the device is in state one indeed under quite conservative condition, where $\alpha$ and $\beta$ proportional coefficients. Likewise, we have

$$
\begin{aligned}
\tau_{x 2(\infty)} & =\gamma \mathrm{MTTF}_{11}+\beta \mathrm{MTTF}_{1} \\
\tau_{x 3(\infty)} & =\gamma \mathrm{MTTF}_{11}+\alpha \mathrm{MTTF}_{21}
\end{aligned}
$$

Under steady-state, the system indexes: $x_{1}(t)=x_{1}(\infty), \quad x_{2}(t)=x_{2}(\infty)$, and $x_{3}(t)=x_{3}(\infty)$, and as well as $x_{1}(\infty)+x_{2}(\infty)+x_{3}(\infty)=1$, must be met, and so it is easy to know $\alpha=x_{2}(\infty), \beta=x_{3}(\infty)$, and $\gamma=$ $x_{1}(\infty)$. And so (28), (29), (30) are proven. Since $\tau_{x 1(\infty)}, \tau_{x 2(\infty)}$ and $\tau_{x 3(\infty)}$ respectively is the expected steady state time of each state, and then the total expected survival time are the their sum for single device, then, the (31) is proven, immediately. (end)

Let $C_{A 1}$ be the cost of each device, and $C_{M 1} \times \tau_{x 1(\infty)}$ be the desired stored cost where $C_{M 1}$ expresses the maintenance cost per unit time, and $C_{U 1} \times \tau_{x 2(\infty)}$ be the desired operating earnings where $C_{U 1}$ expresses the operating earnings per unit time, and $C_{R 1} \times \tau_{x 3(\infty)}$ be the desired PM cost where $C_{R 11}$ expresses the PM cost per unit time, and $C_{N 1} \times N_{1}$ be the desired repairing cost where $N_{1}$ is the expected repairing number on the scope of zero to $t$ and $C_{N 1}$ is the repairing cost per times. And then, the gross expected cost in range of zero to $t$ is

$$
C_{1}=C_{A 1}+\frac{\left(C_{M 1} \times \tau_{x 1(\infty)}-C_{U 1} \times \tau_{x 2(\infty)}+C_{R 1} \times \tau_{x 3(\infty)}+C_{N 1}\right) \times t}{\tau_{1}}
$$

Assuming that the number of the devices is $n$ that must be functioning during steady state, then it is necessary to possess the devices number at least below. 


$$
n_{1}=\frac{n}{x_{2}(\infty)}=n+\frac{x_{1}(\infty)}{x_{2}(\infty)} n+\frac{x_{3}(\infty)}{x_{2}(\infty)} n
$$

Lemma1 (TBM model I) [16] Under TBM, the optimal detecting times per unit time of the device can be written by

$$
u^{*}=\sqrt{\frac{c T_{b}}{T_{i}}}
$$

where $T_{\mathrm{b}}$ means the required downtime time of the device malfunction, and $T_{\mathrm{i}}$ is the required downtime time when the device is detected under PM, and $c$ is a coefficient related to specific function device.

Under the role of (33), the gross downtime time of the device may arrive at the shortest per unit time, which can be described as

$$
T D T^{*}=2 \sqrt{c T_{i} T_{b}}
$$

Proof. The gross downtime time of the device per unit time can be written by

$$
T D T=u T_{i}+\frac{c T_{b}}{u}
$$

Solving the derivative on $u$ on both sides of (35), we then have

$$
\frac{\mathrm{d} T D T}{\mathrm{~d} u}=T_{i}-\frac{c T_{b}}{u^{2}}
$$

Let the left side of the above formula equal zero, and (33) is obtained. Then substituting (33) into (35) and (34) is obtained, immediately.

Lemma2 (TBM model II) [16] Under TBM, the optimal detecting times per unit time of the device can be written by

$$
u^{*}=\ln \left[\frac{f \theta\left(p+C_{N 1}\right)}{\mu\left(p+C_{R 1}\right)}\right]
$$

where $f$ is the device failure rate as $u=0$, and $1 / \theta$ is the average of the detection times following the exponential distribution, and $p$ is the achieved profit per unit time without downtime, and the definitions on $C_{N 1}$ and $C_{R 1}$ are as the former. Under the role of (36), the gross profit per unit time is seen as the largest.

Proof. The profit of the device per unit time can be described as

$$
P R=p-\frac{p u}{\theta}-\frac{p \lambda(u)}{\mu}-\frac{u C_{R 1}}{\theta}-\frac{C_{N 1} \lambda(u)}{\mu}
$$

where $u$ is the detection rate per unit time, and $\mu$ is the repairing rate per unit time, and $\lambda(u)$ is the device failure rate equivalent to $f e^{-u}$.

Let

and (36) is obtained at once. (end)

$$
\frac{\mathrm{d} P R}{\mathrm{~d} u}=0
$$

Definition1 (Passive Maintenance) As the system described by (4) to (8), let the detecting rate $u_{1}$ and $u_{2}$ equal zero, respectively, then the achieved system is just PM model.

Based on Definition1, we can see that the relative consequences and theorems are still be used on TBM by PM, the only difference is that the checking rate of the former is constant, and zero is the latter. 


\section{CBM model Analysis}

CBM is a kind of proactive maintenance mode, and implements maintenance based on the practical demands of the devices, and theoretically is an optimal maintenance activity. In CBM model, considering the checking rates $u_{1}$ and $u_{2}$ being variables, and so we use $u_{1}(\mathrm{t})$ and $u_{2}(\mathrm{t})$ to replace them in TPM mode. And then the formula (10) and (12) become as

$$
\left\{\begin{array}{l}
\mathrm{d} x_{1}(t) / \mathrm{d} t=-\left(\lambda_{2}+v_{1}+\rho_{1} \mu_{1}\right) x_{1}(t)+\left(\lambda_{1}-\rho_{1} \mu_{1}\right) x_{2}(t)-u_{1}(t) x_{1}(t)+\rho_{1} \mu_{1} \\
\mathrm{~d} x_{2}(t) / \mathrm{d} t=\left(\lambda_{2}-\mu_{2}\right) x_{1}(t)-\left(\lambda_{1}+v_{2}+\left(1-\rho_{1}\right) \mu_{2}\right) x_{2}(t)-u_{2}(t) x_{2}(t)+\left(1-\rho_{1}\right) \mu_{2}
\end{array}\right.
$$

Writing the equations above as the matrix form, then

$$
\mathrm{d} \boldsymbol{x}(t) / \mathrm{d} t=\boldsymbol{A} x(t)-\boldsymbol{U}(t) \boldsymbol{x}(t)+\boldsymbol{\mu}=\boldsymbol{A} \boldsymbol{x}(t)-\boldsymbol{X}(t) \boldsymbol{u}(t)+\boldsymbol{\mu}
$$

where the definitions on $\boldsymbol{x}(\boldsymbol{t})$, and $\boldsymbol{\mu}$ are same with (13), and

$$
\begin{gathered}
\boldsymbol{A}=\left[\begin{array}{cc}
-d_{1}^{\prime} & a \\
b & -d_{2}^{\prime}
\end{array}\right] \quad \boldsymbol{U}(t)=\left[\begin{array}{cc}
u_{1}(t) & 0 \\
0 & u_{2}(t)
\end{array}\right] \quad \boldsymbol{X}(t)=\left[\begin{array}{cc}
x_{1}(t) & 0 \\
0 & x_{2}(t)
\end{array}\right] \\
d_{1}^{\prime}=\lambda_{2}+v_{1}+\rho_{1} \mu_{1}, a=\lambda_{1}-\rho_{1} \mu_{1}, \\
d_{2}^{\prime}=\lambda_{1}+v_{2}+\left(1-\rho_{1}\right) \mu_{1}, b=\lambda_{2}-\left(1-\rho_{1}\right) \mu_{1} .
\end{gathered}
$$

Clearly, $\boldsymbol{U}(t) \boldsymbol{x}(t)=\boldsymbol{X}(t) \boldsymbol{u}(t)$.

To arrive at the expected steady-state probability, the checking rate below can be selected according to [6].

$$
\left[\begin{array}{l}
u_{1}(t) \\
u_{2}(t)
\end{array}\right]=\left[\begin{array}{l}
\frac{\rho_{1} \mu_{1}+a x_{2}(\infty)}{x_{1}(\infty)}-d_{1}^{\prime} \\
\frac{\left(1-\rho_{1}\right) \mu_{1}+b x_{1}(\infty)}{x_{2}(\infty)}-d_{2}^{\prime}
\end{array}\right]
$$

where $x_{1}(\infty)$, and $x_{2}(\infty)$ are the expected steady-state probabilities, which satisfy

$$
\begin{aligned}
& 0 \leq x_{1}(\infty) \leq 1, \quad 0 \leq x_{2}(\infty) \leq 1, \quad 0 \leq x_{1}(\infty)+x_{2}(\infty) \leq 1, \\
& \rho_{1} \mu_{1}+a x_{2}(\infty)-d_{1}^{\prime} x_{1}(\infty)>0, \quad \rho_{2} \mu_{2}+b x_{1}(\infty)-d_{2}^{\prime} x_{2}(\infty)>0 .
\end{aligned}
$$

And then, based on same method in Throrem1, we may obtain the $\mathrm{MTTF}_{2}$ of the system (38) as follows.

where

$$
\mathrm{MTTF}_{2}=\mathrm{MTTF}_{21}+\mathrm{MTTF}_{22}
$$

$$
\begin{aligned}
& \operatorname{MTTF}_{21}=\frac{\left(d_{2}^{\prime}+\left(1-\rho_{1}\right) \mu_{1}\right)\left(x_{1}(0)-x_{1}(\infty)\right)+a\left(x_{2}(0)-x_{2}(\infty)\right)}{\left(d_{1}^{\prime}+\rho_{1} \mu_{1}\right)\left(d_{2}^{\prime}+\left(1-\rho_{1}\right) \mu_{1}\right)-a b} \\
& \operatorname{MTTF}_{22}=\frac{b\left(x_{1}(0)-x_{1}(\infty)\right)+\left(d_{1}^{\prime}+\rho_{1} \mu_{1}\right)\left(x_{2}(0)-x_{2}(\infty)\right)}{\left(d_{1}^{\prime}+\rho_{1} \mu_{1}\right)\left(d_{2}^{\prime}+\left(1-\rho_{1}\right) \mu_{1}\right)-a b}
\end{aligned}
$$

And so, according to Theorem3, we still may compute the gross expected cost $C_{2}$ in range of zero to $t$ and the devices number $n_{2}$ at steady-state below

$$
\begin{gathered}
C_{2}=C_{A 2}+\frac{\left(C_{M 2} \times \tau_{x 1(\infty)}-C_{U 2} \times \tau_{x 2(\infty)}+C_{R 2} \times \tau_{x 3(\infty)}+C_{N 2}\right) \times t}{\tau_{2}} \\
n_{2}=\frac{n}{x_{2}(\infty)}=n+\frac{x_{1}(\infty)}{x_{2}(\infty)} n+\frac{x_{3}(\infty)}{x_{2}(\infty)} n
\end{gathered}
$$




\section{Redundant Maintenance Model}

The description is basically same with TBM on redundant part maintenance model, the solely difference lies in that the functional device is given a redundant part as shown Figure 4.

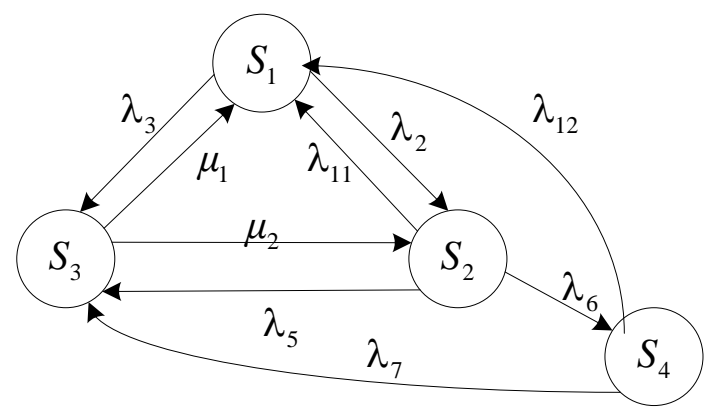

\section{Figure 4. State transition diagram with redundant component}

In Figure 4, the functional state is divided into two parts, where $S_{2}$ expresses that one device is working and another one is spare, and $S_{4}$ represents the working device broken while spare one being working.

According to Figure 4, we may write out state transition equations below.

$$
\left\{\begin{array}{l}
\mathrm{d} x_{1}(t) / \mathrm{d} t=-\left(\lambda_{2}+\lambda_{3}\right) x_{1}(t)+\lambda_{11} x_{2}(t)+\lambda_{12} x_{4}(t)+\mu_{1} x_{3}(t) \\
\mathrm{d} x_{2}(t) / \mathrm{d} t=\lambda_{2} x_{1}(t)-\left(\lambda_{11}+\lambda_{5}+\lambda_{6}\right) x_{2}(t)+\mu_{2} x_{3}(t) \\
\mathrm{d} x_{3}(t) / \mathrm{d} t=\lambda_{3} x_{1}(t)+\lambda_{5} x_{2}(t)+\lambda_{7} x_{4}\left(t-\left(\mu_{1}+\mu_{2}\right) x_{3}(t)\right. \\
\mathrm{d} x_{4}(t) / \mathrm{d} t=\lambda_{6} x_{2}\left(t-\left(\lambda_{12}+\lambda_{7}\right) x_{4}(t)\right. \\
x_{1}(t)+x_{2}(t)+x_{3}(t)+x_{4}(t)=1
\end{array}\right.
$$

Considering that $x_{3}(t)=1-\left[x_{1}(t)+x_{2}(t)+x_{4}(t)\right]$, and then we have

$$
\frac{\mathrm{d} \boldsymbol{x}(t)}{\mathrm{d} t}=\boldsymbol{A}_{2} \boldsymbol{x}(t)+\boldsymbol{\mu}
$$

where

$$
\begin{aligned}
& A_{2}=\left[\begin{array}{ccc}
-d_{11}^{\prime} & a_{1} & a_{2} \\
b & -d_{22}^{\prime} & -\mu_{2} \\
0 & \lambda_{6} & -d_{33}
\end{array}\right], \quad \boldsymbol{x}(t)=\left[\begin{array}{c}
x_{1}(t) \\
x_{2}(t) \\
x_{4}(t)
\end{array}\right], \quad \boldsymbol{\mu}=\left[\begin{array}{c}
\mu_{1} \\
\mu_{2} \\
0
\end{array}\right] \\
& d_{11}^{\prime}=\lambda_{2}+\lambda_{3}+\mu_{1}, \quad a_{1}=\lambda_{11}-\mu_{1}, \quad a_{2}=\lambda_{12}-\mu_{1} \\
& b=\lambda_{2}-\mu_{2}, \quad d_{22}^{\prime}=\lambda_{11}+\lambda_{5}+\lambda_{6}+\mu_{2}, \quad d_{33}=\lambda_{12}+\lambda_{7}
\end{aligned}
$$

As $t \rightarrow \infty$, the steady-state probabilities of the system is 


$$
\left.\begin{array}{c}
x_{1}(\infty)=\frac{\left(d_{22}^{\prime} d_{33}+\lambda_{6} \mu_{2}\right) \mu_{1}+\left(a_{1} d_{33}+a_{2} \lambda_{6}\right) \mu_{2}}{-d_{11}^{\prime} d_{22}^{\prime} d_{33}+a_{2} b_{1} \lambda_{6}-d_{11}^{\prime} \lambda_{6} \mu_{2}+d_{33} a_{1} b_{1}} \\
x_{2}(\infty)=\frac{b_{1} d_{33} \mu_{1}+d_{11}^{\prime} d_{33} \mu_{2}}{-d_{11}^{\prime} d_{22}^{\prime} d_{33}+a_{2} b_{1} \lambda_{6}-d_{11}^{\prime} \lambda_{6} \mu_{2}+d_{33} a_{1} b_{1}} \\
x_{4}(\infty)=\frac{-b_{1} \lambda_{6} \mu_{1}+d_{11}^{\prime} \lambda_{6} \mu_{2}}{-d_{11}^{\prime} d_{22}^{\prime} d_{33}+a_{2} b_{1} \lambda_{6}-d_{11}^{\prime} \lambda_{6} \mu_{2}+d_{33} a_{1} b_{1}} \\
x_{3}(\infty)=1-x_{1}(\infty)-x_{2}(\infty)-x_{4}(\infty)
\end{array}\right\}
$$

Then, using identical method in Throrem1, we may obtain the $\mathrm{MTTF}_{3}$ of the system (42) as follows.

where

$$
\mathrm{MTTF}_{3}=\mathrm{MTTF}_{31}+\mathrm{MTTF}_{32}
$$

$$
\begin{aligned}
\operatorname{MTTF}_{31} & =\frac{\bar{d}_{2} d_{33}\left(x_{1}(0)-x_{1}(\infty)\right)+\bar{a} d_{33}\left(x_{2}(0)-x_{2}(\infty)\right)+d\left(x_{4}(0)-x_{4}(\infty)\right)}{d_{33}\left(d_{1} \bar{d}_{2}-\bar{a} b\right)} \\
\operatorname{MTTF}_{32} & =\frac{\bar{d}_{3} b\left(x_{1}(0)-x_{1}(\infty)\right)+\bar{d}_{3} d_{1}\left(x_{2}(0)-x_{2}(\infty)\right)+\bar{b}\left(x_{4}(0)-x_{4}(\infty)\right)}{d_{33}\left(d_{1} \bar{d}_{2}-\bar{a} b\right)}
\end{aligned}
$$

where

$$
\begin{aligned}
& \bar{d}_{2}=d_{22}^{\prime}+\frac{\lambda_{6}}{d_{33}} \mu_{2}, \quad \bar{a}=a_{1}+\frac{\lambda_{6}}{d_{33}} a_{2}, \quad e=-a_{2} \bar{d}_{2}, \quad d_{1}=\lambda_{2}+\lambda_{3}+\mu_{1}, \\
& \bar{b}=d_{11}^{\prime}\left(\bar{d}_{2}-\mu_{2}\right)-b\left(\bar{a}-a_{2}\right), \bar{d}_{3}=d_{33}+\lambda_{6} .
\end{aligned}
$$

And so, according to Theorem 3 , we can compute the gross expected cost $C_{3}$ in range of zero to $t$ and the devices number $n_{3}$ at steady-state below

$$
\begin{gathered}
C_{3}=C_{A_{3}}+\frac{\left(C_{M 3} \times \tau_{x 1(\infty)}-C_{U 3} \times \tau_{x 2(\infty)}+C_{R 3} \times \tau_{x 3(\infty)}+C_{N 2}\right) \times t}{\tau_{3}} \\
n_{3}=\frac{n}{x_{2}(\infty)+x_{4}(\infty)}=n+\frac{x_{1}(\infty)}{x_{2}(\infty)+x_{4}(\infty)} n+\frac{x_{3}(\infty)}{x_{2}(\infty)+x_{4}(\infty)} n
\end{gathered}
$$

\section{Examples}

A production enterprise requires 500 sets of devices operating to satisfy the market demands at ordinary times. To ensure the stability of the production process, the enterprise implements the preventive maintenance for these cells, and arranges a checking-repairing centre and spare parts warehouse. To compare the maintenance property indexes of each model, a numerical computation is made based on the supplied real data of the enterprise as follows.

In passive maintenance model, the transfer rates per month of the parameters are described by $\lambda_{1}=1.5625, \lambda_{2}=1.875, \lambda_{3}=v_{1}=0.9375, \lambda_{4}=v_{2}=0.9375, \mu_{1}=1.8750, \rho_{1}=2 / 3, u_{1}=0$, and $u_{2}=0$, and the initial state probabilities satisfy $x_{1}(0)=1 / 2, x_{2}(0)=1 / 2, x_{3}(0)=0$, and as well as cost data $C_{\mathrm{A} 1}=C_{\mathrm{A}}, C_{\mathrm{M} 1}=0.01 C_{\mathrm{A}}, C_{\mathrm{U} 1}=10 C_{\mathrm{A}}, C_{\mathrm{R} 1}=0.1 C_{\mathrm{A}}, C_{\mathrm{N} 1}=0.5 C_{\mathrm{A}}$. Firstly, we may work out the 
expected steady probabilities based on (17) to (19), i.e., $x_{1}(\infty)=1 / 3, x_{2}(\infty)=1 / 3$, and $x_{3}(\infty)=1 / 3$. Then from Theorem1 to Theorem3, and as well as (31), the investment payback period can be worked out as $T_{1}=3.5$ months by letting $C_{1}=0$, and the required gross device number is $n_{1}=1500$ from $(32)$.

In TBM model, what we require to do is to correct the parameters $\lambda_{3}$ and $\lambda_{4}$ due to PM intervention on the basis of passive maintenance model, i.e., $\lambda_{3}=u_{1}+v_{1}, \lambda_{4}=u_{2}+v_{2}$. For TBM model I, based on descriptions on Figure 2, in Lemma1, let $T_{\mathrm{b}}=T_{\mathrm{i}}$, and $c=3$ for the stored devices and $c=2$ for the operating ones. Thus, according to (33), we may work out $u_{1}=1.732$, and $u_{2}=1.414$, and then, likewise, we can work out $T_{1}=0.54$ months, and $n_{1}=2203$ from (31) and (32). For another TBM model II, based on (36) in Lemma2, we let $f=v_{1}$ and $1 / \theta=\rho_{1} \mu_{1}$ and $u_{1}$ can be computed and $f=v_{2}$ and $1 / \theta=\left(1-\rho_{1}\right) \mu_{1}$ and $u_{2}$ can be computed, and then order $\lambda_{3}=u_{1}+v_{1}, \lambda_{4}=u_{2}+v_{2}$. And then, in the same way, we can work out $T_{1}=0.5$ months, and $n_{1}=1953$.

In CBM model, what we require to concern is an expected steady state probability that should be designed in advance, and also the parameters $C_{\mathrm{A} 2}=C_{\mathrm{A}}, C_{\mathrm{M} 2}=0.01 C_{\mathrm{A}}, C_{\mathrm{U} 2}=10 C_{\mathrm{A}}$, $C_{\mathrm{R} 2}=0.1 C_{\mathrm{A}}, C_{\mathrm{N} 2}=0.05 C_{\mathrm{A}}$. Due to PM intervention, the steady state probabilities of the devices decrease a bit for the state $S_{1}$ and $S_{2}$ compared with passive maintenance, while the steady state probability of $S_{3}$ increases a bit. We might as well let $x_{1}(\infty)=1 / 4, x_{2}(\infty)=1 / 4$, and then from (39), we can work out $u_{1}$ and $u_{2}$, and meantime order $\lambda_{3}=u_{1}+v_{1}, \lambda_{4}=u_{2}+v_{2}$. And then applying the same way, we obtain $T_{2}=0.33$ months, and $n_{2}=2000$.

In redundant maintenance model, we need to supply further the parameter values with $\lambda_{5}=0.46875, \lambda_{6}=1.2500, \lambda_{7}=0.46875, \lambda_{11}=0.78125, \lambda_{12}=0.78125$, and as well as $C_{\mathrm{A} 3}=2 C_{\mathrm{A}}$, $C_{\mathrm{M} 3}=0.01 C_{\mathrm{A}}, C_{\mathrm{U} 3}=10 C_{\mathrm{A}}, C_{\mathrm{R} 3}=0.1 C_{\mathrm{A}}$, and $C_{\mathrm{N} 3}=0.5 C_{\mathrm{A}}$ on the basis of passive maintenance, and then using uniform method, based on (44) and (45), we can obtain $T_{3}<0$ months, and $n_{3}=1000$, which means that the investment can not be paid back.

Clearly, the investment payback period is the shortest using CBM model of 0.33 months alone and approximately 10 day, and 0.64 months for TBM model I and 0.5 months for TBM model II, and also 3.5 months for passive maintenance. For redundant maintenance model, the investment can not be paid back. It is noted that the repairing cost in CBM and TBM is relatively low compared with ones in passive and redundant maintenance model, the reason is that the PM cost is lower than corrective maintenance cost. The computing results also show that the two kinds of TBM models almost have same results, and their investment payback period is about 0.5 months and approximately 15 days.

\section{Conclusion}

The several significant maintenance management models are presented on the equipments operation in this paper, and whose life cycle operating characteristics are analyzed and stead state probabilities are calculated. Based on it, the paper mainly implements investment cost and profits analysis on them, and evaluates their advantages and flaws, and thus provides operation maintenance management consults for the devices. The presented models mentioned in this paper not only can be used to instruct the production business practice for the enterprises, but also give out the evaluation for their preventive maintenance projects. The model proposed in the paper can better guide provision, maintenance, and reverse plan of the devices, and possesses broad application prosperity. 


\section{Acknowledgements}

This project is supported by the National Natural Foundation of China (Grant No.61263004) and Gansu Province Natural Science Foundation (Grant No.1212RJZA071).

\section{References}

[1] P. A. Scarf, "A framework for condition monitoring and condition based maintenance", Quality Technology and Quantitative Management, vol. 4, no. 2, (2007), pp. 301-312.

[2] R. Jiang and D. N. P. Murthy, "Maintenance Decision Models for Management", Science Press, Beijing, (2008).

[3] D. N. Khandelwal, J. Sharma and L. M. Ray, "Optimal periodic maintenance of a machine", IEEE Transactions on Automatic Control, vol. 24, no. 3, (1979), pp. 513-513.

[4] Nguyen and Murthy, "Optimal preventive maintenance policies for repairable system", IEEE Transactions on Reliability, vol. 26, no. 3, (1981), pp. 1181-1194.

[5] B. W. Li, "New Thoughts and New Modes on Equipment Management", Mechanical Industry Press, Beijing, (2010).

[6] H. S. Su, "Control strategy on preventive maintenance of repairable device", Journal of Zhejiang University (Engineering Science), vol. 44, no. 7, (2010), pp. 136-145.

[7] H. S. Su, "Reliability and security analysis on 3-vote-2 voting System", International Journal of Security \& Its Applications, vol. 7, no. 4, (2013), pp. 327-334.

[8] M. Rausand, "System Reliability Theory: Models", Statistics Methods and Application (2nd Edition), Wiley, New Jersery, (2004).

[9] W. M. Goble, "Control System Safety Evaluation and Reliability", ISA, Raleigh, (2010).

[10] W. Wang, "A multi-component and multi-failure mode inspection model based on delay time concept", Reliability Engineering and System Safety, vol. 95, (2010), pp. 912-950.

[11] J. Cao and G. Chen, "An introduction to reliability mathematics", Higher Education Press, Beijing, (2006).

[12] H Su and Y. Zhang, "Non-Markov repairable model analysis on two modular redundant systems", Chinese Journal of Applied Probability and Statistics, vol. 24, no. 2, (2008), pp. 166-174.

[13] G. Gong and M. Qian, "Applied Random Process", Tsinghua University Press, Beijing, (2004).

[14] J. Sheng, C. Xie and C. Pan, "Probability Theory and Mathematical Statistics", Zhejiang University Press, Hangzhou, (1996).

[15] D. Zheng, "Linear Control Theory", Tsinghua University Publishing House, Beijing, (2002).

[16] H. Zuo, J Cai and H. Wang, "Maintenance Decision Theory and Method", Aviation Industry Press, Beijing, (2008).

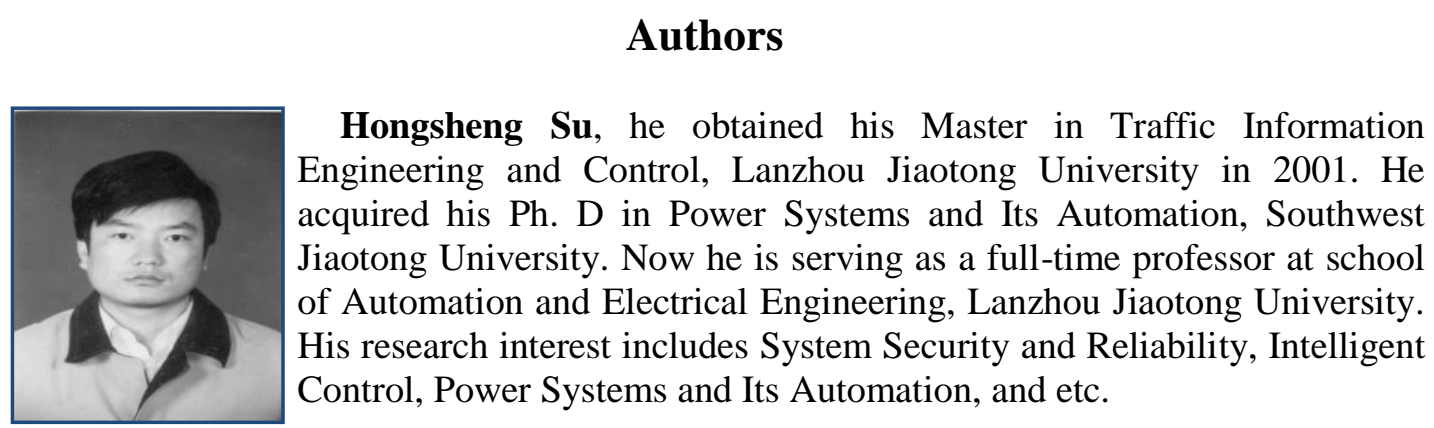

